CLAWAR 2018: 21st International Conference on Climbing and Walking Robots and the Support Technologies for Mobile Machines, Panama City, Panama, 10-12 September 2018

\title{
REHABILITATION PROTOTYPE TO IMPROVES MOBILITY OF ACUTE ROTATOR CUFF INJURY
}

\author{
MARIANA TORRENTE ROCHA, LAURA JIMÉNEZ GUZMÁN \\ El Bosque University, Bioengineering Department \\ Av. Cra 9 No. 131 A-02 Bogota, Colombia \\ HERNANDO LEÓN-RODRÍGUEZ \\ El Bosque University, Electronics Engineering and Bioengineering Department \\ Av. Cra 9 No. 131 A-02 Bogota, Colombia
}

The deterioration of the components of the rotator cuff is one of the most frequent causes of musculoskeletal pain and disability in the world. The conditions of the rotator cuff increase with the passage of time, since they have a direct relationship with a process of deterioration rather than with a traumatic event. In order to decrease pain and recover shoulder movement, patients are usually treated by regular sessions with a physiotherapist. However, more sessions are required than those stipulated to fully obtain recover mobility This paper present the development of portable prototype to rehabilitate acute rotator cuff injure, designed from biomechanical analysis and ranges the kinematics and dynamics articulated in the present design determine important requirements in obtaining greater functionality of the prototype and with it, allows the identification of sensors and actuators for executing two types of specific movements: flexion / extension and external / internal rotation by means of a soft rehabilitation robot

\section{Introduction}

The component's deterioration of the rotator cuff is one of the most frequent causes of musculoskeletal pain and disability in the world. The incidence of shoulder pain in the general population is around 11.2 cases per 1,000 patients per year. It is considered to be the most prevalent soft tissue pathology, with an estimated incidence of rotator cuff injuries of 3.7 per 100,000 per year.

The pathology of the rotator cuffs is associated with the overuse of the joint by work, sports, avascularity, mechanical failure located in the supraspinatus tendon, ruptures caused by deterioration or even by the entrapment that the tendon suffers between the humerus and the acromion. There are other risk factors that can lead to a rotator cuff injury, such as obesity, hypercholesterolemia, genetic factors, anatomical variations, scapular dyskinesia and glenohumeral instability. [1]

Currently, the rehabilitation of rotator cuffs starts from the application of conventional therapies, to the use of robotics as alternative mechanisms, which integrate engineering concepts such as compensation of force, motor control at speeds calibrated according to the motor functions of each individual and sensory contributions that through the repetition of exercises in intensive training 
programs manage to recover mobility limitations.[2] [10] Some of the referents are Armeo Spring who by providing support for the weight of the arm, allows patients to use any remaining motor function and encourages them to reach a greater number of reach and grip movements based on specific therapeutic objectives, from an arm grab exoskeleton [3]; In-Motion Shoulder-Elbow Robot allows to quantify the control of the motor of the upper extremities and with the recovery of the movement, which allows the doctors to distinguish the real recovery from the compensation of the patient [4]; shoulder exoskeletons; CableRobots who have very good kinematic and dynamic characteristics, and also show other properties such as: portability and economy of costs, which also make them suitable for medical applications and rehabilitation even though based on the physical nature of the cables that can only pull and not push [5]; isokinetic force machines such as Humac Norm Testing and Rehabilitation System that allows the diagnosis and treatment of the performance of muscles and joints of orthopaedic patients, starting from the principle of isokinetic force, under the correct anatomical positioning and a positive stabilization to examine the musculature that surrounds the shoulder [6]. Based on the aforementioned developments, the new technologies point to the use of soft materials, which improve the ergonomics of the rehabilitation system like active soft orthotic system [7] [8] [9]

\section{Biomechanical evaluation of patient}

The biomechanics establishes a specific protocol to certain angles according to each movement as a pattern of healthy patients as example: the flexion motion goes from $0^{\circ}$ to $180^{\circ}$, the extension motion from $0^{\circ}$ to $60^{\circ}$ for the sagittal plane; adduction from $0^{\circ}$ to $45^{\circ}$, abduction from $0^{\circ}$ to $180^{\circ}$ in the frontal plane; the flexion of $130^{\circ}$ to $5^{\circ}$, extension of $40^{\circ}$ to $50^{\circ}$ with respect to the horizontal plane; external rotation from $0^{\circ}$ to $40^{\circ}$ to $60^{\circ}$ and internal rotation from $0^{\circ}$ to $90^{\circ}$; and external and internal movements, both up to $70^{\circ}$. Although the biomechanical analysis allows limit the total movement angles of the articulation, the functional ranges of mobility are the representatives of movement with minimum balance in the comfortable execution of daily activities. From this conditions, the mechanism of operation of the prototype is designed, being specific in movements of flexoextension (A), and internal and external rotation of the Shoulder (B).

\section{Concepts of prototype design}

The figure 1 is showing the design of a portable system driven by two gearmotors. The kinematic and dynamic analyses are based on two of the movements adapted by the articulation: flexion / extension, external / internal rotation which activate two degrees of freedom respectively. The prototype consists of two supports for the arm and the forearm, which will maintain the extremity in the ranges of joint movements required to perform the different rehabilitation exercises. [11] [12] [13] 


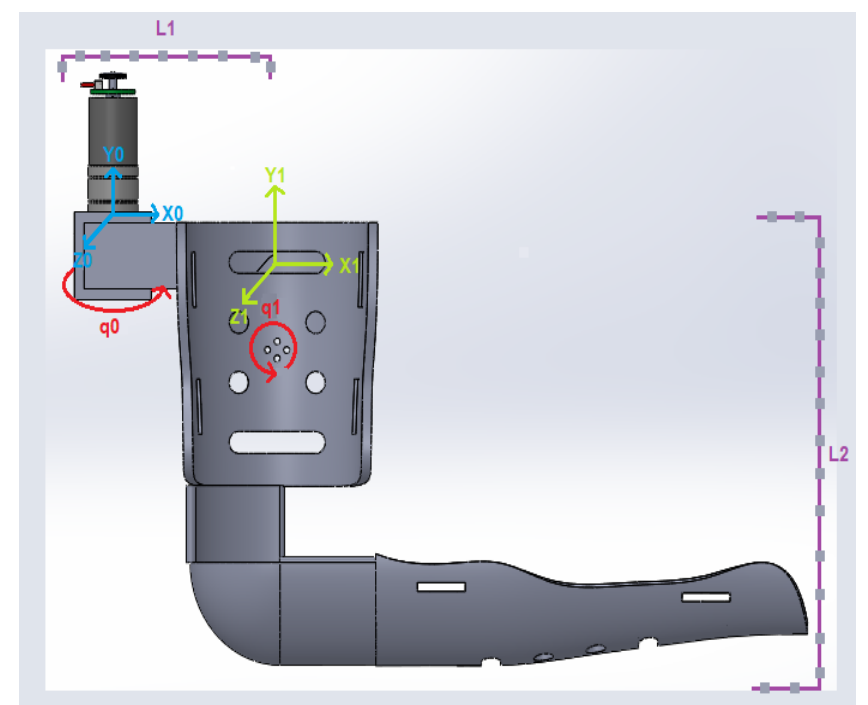

Figure 1: Design of shoulder rehabilitation robot first and second joint.

\subsection{Kinematic Design}

The kinematic system was obtained from a reference axis of the shoulder, which was proposed for the design of the system in the arm. (See figure 1)

$$
\boldsymbol{R}_{\mathrm{Y}, \mathrm{q} 0}=\left(\begin{array}{ccc}
\cos (\mathrm{q} 0) & 0 & \operatorname{sen}(\mathrm{q} 0) \\
0 & 1 & 0 \\
\operatorname{sen}(\mathrm{q} 0) & 0 & \cos (\mathrm{q} 0)
\end{array}\right) \operatorname{Tras}_{z, l 1}=\left[\begin{array}{cccc}
1 & 0 & 0 & 0 \\
0 & 1 & 0 & 0 \\
0 & 0 & 1 & l 1 \\
0 & 0 & 0 & 1
\end{array}\right]
$$

The rotation matrix RY, q0 gives the projection of the coordinates in two coordinate systems. the equation 1 is the first rotation matrix it is evident that the system rotates on the $\mathrm{Y}$ axis and the articulation that rotates on this axis is the one called q0. In the translation matrix Tras z, we can see which link is transferred for the first system and on which axis the movement is observed. In this case, link 1 moves on the $\mathrm{z}$ axis.

$$
A_{0}^{1}=\left[\begin{array}{cccc}
c_{q 0} & 0 & s_{q 0} & 0 \\
0 & 1 & 0 & 0 \\
-s_{q 0} & 0 & c_{q 0} & -l 1 *^{*} s_{q 0} \\
0 & 0 & 0 & 1
\end{array}\right]
$$

The equation 2 is the homogenous transformation matrix A01, we can see the projection of system 0 in system 1 and we can see the sum or product point between the matrices of rotation and translation. The breasts and cosines that are evident in the rotation matrix, are the projections of the vectors in both $\mathrm{x}$ and $\mathrm{y}$. 


$$
R_{\mathrm{Z}, \mathrm{q1}}=\left(\begin{array}{ccc}
\cos (\mathrm{q} 1) & -\operatorname{sen}(\mathrm{q} 1) & 0 \\
\operatorname{sen}(\mathrm{q} 1) & \cos (\mathrm{q} 1) & 0 \\
0 & 0 & 1
\end{array}\right) \quad \operatorname{Tras}_{x, 12}=\left|\begin{array}{cccc}
1 & 0 & 0 & l 2 \\
0 & 1 & 0 & 0 \\
0 & 0 & 1 & 0 \\
0 & 0 & 0 & 1
\end{array}\right|
$$

The rotation matrix RZ, q1 gives the projection of the coordinates in two coordinate systems. In the first rotation matrix it is evident that the system rotates on the $\mathrm{Z}$ axis and the articulation that rotates on this axis is the one called q1. In the translation matrix Tras $x_{x, 12}$ you can see which link is transferred to the second system and on which axis the movement is observed. In this case, link 2 moves on the $\mathrm{x}$-axis.

$$
A_{1}^{2}=\left[\begin{array}{cccc}
c_{q 1} & -s_{q 1} & 0 & 12 * c_{q 1} \\
s_{q 1} & c_{q 1} & 0 & 12 * s_{q 1} \\
0 & 0 & 1 & 0 \\
0 & 0 & 0 & 1
\end{array}\right]
$$

In the homogeneous transformation matrix A12, we can see the projection of system 1 in system 2 and we can see the sum or product point between the matrices of rotation and translation.

$$
A_{0}^{2}=\left[\begin{array}{cccc}
c_{q 0} s_{q 1} & -c_{q 0} s_{q 1} & 0 & l 2 * c_{q 1} \\
s_{q 1} & c_{q 1} & 0 & l 2 * s_{q 1} \\
0 & 0 & 1 & -l 1 * s_{q 0} \\
0 & 0 & 0 & 1
\end{array}\right]
$$

After obtaining the homogeneous transformation matrix of the system q1 and $\mathrm{q} 0$, multiplication or cross product of both homogeneous matrices is performed to find the position of the end terminal, with respect to the whole system.

$$
\begin{aligned}
\mathrm{X}=12 * \operatorname{Cos}(\mathrm{q} 1) ;\left(\frac{d}{d t} \mathrm{x}\right) & =\frac{d}{d t}(12 * \operatorname{Cos}(\mathrm{q} 1))=>\mathrm{Vx}=(-\dot{q} 1 * l 2 * \operatorname{Sen}(q 1)) \\
\mathrm{Y}=12 * \operatorname{Sen}(\mathrm{q} 1) ;\left(\frac{d}{d \mathrm{y}} \mathrm{y}\right) & =\frac{d}{d t}(12 * \operatorname{Sen}(\mathrm{q} 1)) \Rightarrow \mathrm{Vy}=(\dot{q} 1 * l 2 * \operatorname{Cos}(q 1)) \\
\mathrm{Z}=11 * \operatorname{Sen}(\mathrm{q} 0) ;\left(\frac{d}{d t} \mathrm{z}\right) & =\frac{d}{d t}(11 * \operatorname{Sen}(\mathrm{q} 0)) \Rightarrow>\mathrm{Vz}=(\dot{q} 0 * l 1 * \operatorname{Cos}(q 0)) \\
{\left[\begin{array}{c}
\dot{x} \\
\dot{y} \\
\dot{z}
\end{array}\right] } & =\left[\begin{array}{ccc}
0 & -l 2 * \operatorname{sen}(q 1) & 0 \\
0 & l 2 * \cos (q 1) & 0 \\
l 1 * \cos (q 0) & 0 & 0
\end{array}\right]\left[\begin{array}{l}
q 0 \\
q 1 \\
q 2
\end{array}\right]
\end{aligned}
$$

The Jacobian matrix is used to determine the speed of movement in each of the axes and also to determine the kinetic energy, it was necessary to calculate the velocity vector, which is composed of the sum between the vector derivative of $\mathrm{r}_{\mathrm{CM}}$ center of translational mass plus the angle of rotation, by the cross product of the vector $\mathrm{r}_{\mathrm{CM}}$ of center of mass. Once the velocity vector is found, the kinetic energy and the potential energy are found.

$$
\begin{gathered}
\overrightarrow{\boldsymbol{v}}=\dot{\vec{r}}_{C M} \text { traslacional } \\
\mathrm{K}=\frac{1}{2} m|\overrightarrow{\boldsymbol{v}}|^{2}+\frac{1}{2} \omega^{T} I \omega
\end{gathered}
$$




$$
u=\mathrm{mgh}=0
$$

The vector $r_{1}$ is composed of a medium of link 1 in the direction $i 0$, while the vector $\mathrm{w} 1$ is given by the rotation $\mathrm{q} 0$ in the opposite direction to the movement $\mathrm{j}_{0}$, the vector $\mathrm{v}_{1}$ is given by a means of link 1 by the rotation $\mathrm{q} 0$ in the address $\mathrm{k}$.

$$
\begin{aligned}
& \overrightarrow{r 1}=\frac{l 1}{2}\left(\widehat{i_{0}}\right) \\
& \overrightarrow{w 1}=q_{0}\left(-\hat{\jmath}_{0}\right)
\end{aligned}
$$

After determining each of the variables $(\vec{r} 1 \mathrm{y} \vec{w} 1)$, we find the velocity, which gives us as a result, since the derivative of $\mathrm{R} 1$ with respect to $\mathrm{q} 0$ is 0 .

$$
\overrightarrow{v 1}=\frac{l 1}{2} q_{0}(\hat{k})
$$

Once the velocity is found, the value is replaced in the kinetic energy equation, where WT is the transpose of the vector $\vec{k} 1$ and I is a matrix of inertia.

$$
\mathrm{K} 1=\frac{1}{2} m\left(\frac{l 1}{2} q_{0}\right)^{2}+\frac{1}{2}\left[\begin{array}{c}
0 \\
q_{0} \\
0
\end{array}\right] \mathrm{I}\left[\begin{array}{lll}
0 & q_{0} & 0
\end{array}\right]
$$

In addition to this, the potential energy is found where $\mathrm{m} 1$ corresponds to the mass of the first link and $g$ to the value of gravity.

$$
u=m 1 g \frac{1}{2}
$$

For the second joint articulation of the system showing in figure 1, the same methodology was carried out, with which the energies were obtained in the first articulation. With the difference of the variable $\vec{r} 2$.

$$
\begin{gathered}
\overrightarrow{r 2}=l 1\left(-\hat{\imath}_{0}\right)+\frac{l 2}{2}\left(\hat{k}_{1}\right) \\
\overrightarrow{w 2}=q_{1}\left(\hat{k}_{1}\right) \\
\overrightarrow{v 2}=-q_{1} \frac{l 2}{2}\left(\hat{\imath}_{1}\right)+l 1 q_{1}\left(\hat{U}_{1}\right) \\
\mathrm{K} 2=\frac{1}{2} m 2\left(-q_{1} \frac{l 2}{2}\right)^{2}+\left(l 1 q_{1}\right)^{2}+\frac{1}{2}\left[\begin{array}{c}
0 \\
0 \\
q_{1}
\end{array}\right] \mathrm{I}\left[\begin{array}{lll}
0 & 0 & q_{1}
\end{array}\right] \\
u=m 2 g l 1
\end{gathered}
$$

\subsection{Design and operation characteristics.}

The operation of this prototype starts from a $12 \mathrm{~V}$ power supply that ensures its portability, which replaces the L298N (Bridge H) that will give the control of rotation of two DC motors. Because it is necessary to determine the ranges of extension / flexion movement, external/internal rotation, the angles are sensed by using two encoders, one for each DC motor showing in figure 2 . The control of said DC motors, is given by a software system, Raspberry PI3B, controlled by the Python programming language. A 7805 voltage regulator is required because the software voltage should not exceed $5 \mathrm{~V}$. 


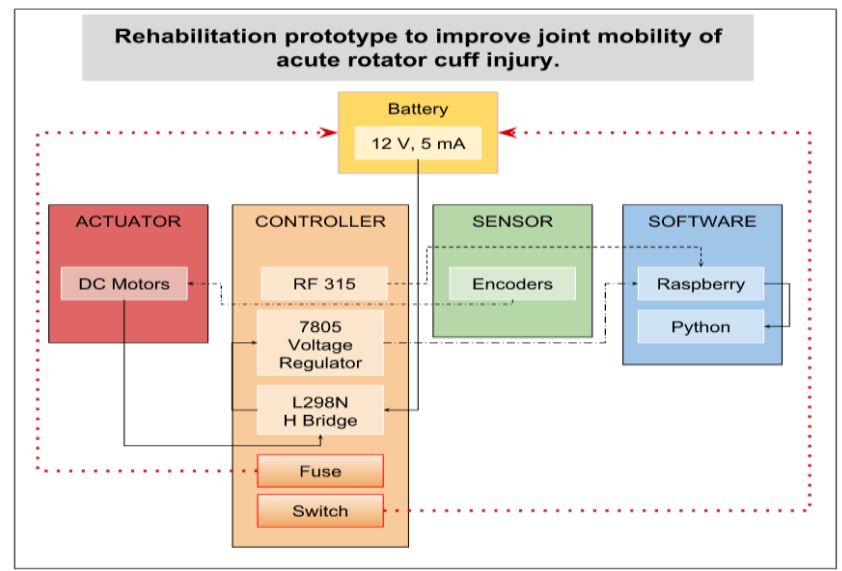

Figure 2: Schematic diagram of control architecture.

\subsection{System Prototype.}

The prototype consists of two stabilizing components showing in figure 3 . The first component consists of a piece that supports the forearm and the arm, and has an elongation fabric that allows the user to have mobility in the elbow (1). The second component is an abdominal stabilizer, which brings stability to the back, and so that the therapy is exercised upright, without complications to the spine, in addition, the trellis has two shoulder straps or stabilizers that they allow the weight of the actuators to dissipate through them, in addition, the trellis has a pocket that groups the electronic components, in order to make it easy to access adjustments or even remove the battery to charge it (2). The actuator that exerts the movement of flexion - extension, has a support that is connected directly to the trellis and a connector that intertwines the other actuator, which performs the external and internal rotation movement and is connected to the forearm support by a piece which lengthens as the flexion movement (4) is performed. Then you can see the two pieces coupled (4, 5 and 6$)$.

\subsection{Sensors and actuator to monitor the patient's position.}

In order to control the movements of the patient and emit four types of therapies, in which, each one contemplates the four movements with different repetitions and angular moments. We use two optical encoders to detect direction and rotation by a beam emitted LED that passes through a code disk for each joint. The actuators used for the prototype are RS-545SH-22100, those have an operating range of 12 to $24 \mathrm{~V}$, an unloaded speed of $4200 \mathrm{rpm}$ at a current of 0.16 A. One of the most important characteristics are the maximum efficiency, since they have a torque of $19.4 \mathrm{mN}-\mathrm{m}$ and a power of $4.36(\mathrm{~W})$. However, since its main function is to support therapy movements, we needed it to have enough 
strength to lift a load like the arm, for this we added two reduction boxes or gearbox of 1:500 in each motor, as a result decreased its speed at its maximum efficiency and increased its strength or torque.

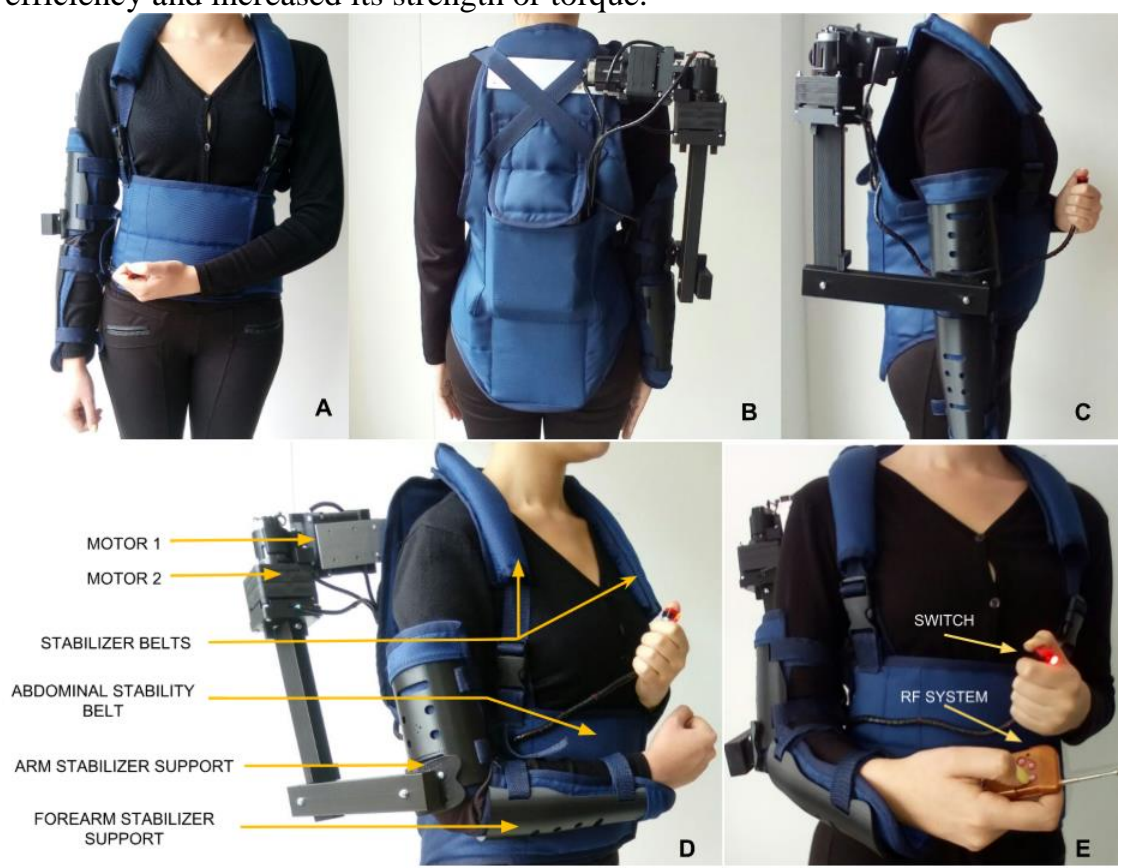

Figure 3: Prototype of shoulder rehabilitation robot system; (A): front, (B): posterior sagittal axis of the device, (C): right view, (D): stabilizing components, (E): emergency switch and control access.

\section{Discussion result and conclusions}

Results are not obtained explicitly related to rotator cuff injury, indispensable parameters are obtained in the operation design of the device, but the functional ranges in which the device responds comfortably and pertinent to a rehabilitation process due to injury, together with the addition of mechanical stops that go to the limits of functional angles.

Once the prototype was built according to the anthropometric measurements, it was possible to demonstrate that it is an ergonomic prototype, since the actuators adapt to the anatomical characteristics of the Glenohumeral joint, because the insertions of the actuators are similar to the anatomical insert that exists between the humerus and the omoplate.

\section{Acknowledgments}

The authors wish to thank César Rocha Libreros, orthopaedic surgeon and reconstructive and knee arthroscopy surgeon of Fundación Cardio Infantil, 


\section{References}

1. Orth T, Paré J, Froehlich JE; Current concepts on the genetic factors in rotator cuff pathology and future implications for sports physical therapists. International Journal of Sports Physical Therapy. 2017;12 (2):273-285.

2. Chiara Sicuri, Giuseppe Porcellini, Giovanni Merolla; Robotics in shoulder rehabilitation; Muscles, Ligaments and Tendons Journal 2014; 4: 207-213.

3. Domien Gijbels, Ilse Lamers, Lore Kerkhofs, Geert Alders, Els Knippenberg, Peter Feys; The Armeo Spring as training tool to improve upper limb functionality in multiple sclerosis: a pilot study; Journal of Neuro Engineering and Rehabilitation 2011, 8:5

4. James Cannan and Huosheng Hu, Upper Body Rehabilitation A Survey; University of Essex, Colchester CO4 3SQ, U.K; ISSN 1744-8050; 2012

5. W. Nunes, L. Rodriguez and L. Oliveira; Cable based parallel manipulator for rehabilitation of shoulder and elbow movements; IEEE Int Conf Rehabil Robot; 2011.

6. Bas Habets, J. Bart Staal, Marsha Tijssen and Robert van Cingel; Intrarater reliability of the Humac NORM isokinetic dynamometer for strength measurements of the knee and shoulder muscles; Res Notes (2018) 11:15.

7. Kesner, S., Jentof, L., Hammond III, F., Howe, R., \& Popovic, M.; Design Considerations for an Active Soft Orthotic System for Shoulder Rehabilitation; 33rd Annual International Conference of the IEEE Engineering in Medicine and Biology Society (EMBC); 2011

8. Ciarán T. O'Neill, Nathan S. Phipps, Leonardo Cappello, Sabrina Paganoni and Conor J. Walsh; A Soft Wearable Robot for the Shoulder: Design, Characterization, and Preliminary Testing; IEEE Int Conf Reh. Robot. 2017.

9. Ignacio Galiana, Frank L. Hammond, Robert D. Howe, and Marko B. Popovic; Wearable Soft Robotic Device for Post-Stroke Shoulder Rehabilitation: Identifying Misalignments; 2012 IEEE/RSJ International Conference on Intelligent Robots and Systems October 7-12, 2012. Vilamoura, Algarve, Portugal.

10. Rodrigo Gonçalves Cerejo Antunes; A Robotic System for Musculoskeletal Rehabilitation of the Shoulder; Biomedical Engineering - U de Lisboa; 2016.

11. Tobias Nef, Marco Guidali and Robert Riener; ARMin III - arm therapy exoskeleton with an ergonomic shoulder actuation; Applied Bionics and Biomechanics Vol. 6, No. 2, June 2009, 127-142.

12. Bongsu Kim and Ashish D Deshpande; An upper-body rehabilitation exoskeleton Harmony with an anatomical shoulder mechanism: Design, modeling, control, and performance evaluation; The International Journal of Robotics Research; 1-22, 2017.

13. Justin Hunt, Hyunglae Lee, Panagiotis Artemiadis; A Novel Shoulder Exoskeleton Robot Using Parallel Actuation and a Passive Slip Interface; Journal of Mechanisms and Robotics; 2017 